\title{
A comprehensive map of genetic interactions in childhood cancer reveals multiple underlying biological mechanisms
}

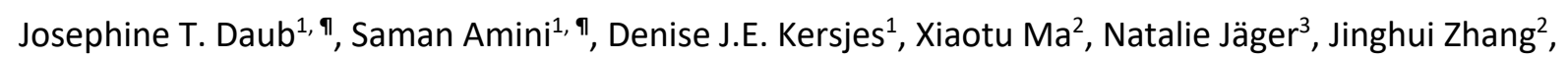
Stefan M. Pfister ${ }^{3,4}$, Frank C.P. Holstege ${ }^{1}$, Patrick Kemmeren ${ }^{1, *}$

${ }^{1}$ Princess Máxima Center for Pediatric Oncology, Utrecht, The Netherlands

${ }^{2}$ Computational Biology, St Jude Children's Research Hospital, Memphis, Tennessee 38105, USA

${ }^{3}$ Hopp Children's Cancer Center Heidelberg (KiTZ) and Division of Pediatric Neurooncology, German Cancer Consortium (DKTK) and German Cancer Research Center (DKFZ), Heidelberg, Germany

${ }^{4}$ Heidelberg University Hospital, Department of Pediatric Hematology and Oncology, Heidelberg, Germany

* Corresponding author

I These authors contributed equally to this work. 
bioRxiv preprint doi: https://doi.org/10.1101/2020.11.17.385120; this version posted December 5,2020 . The copyright holder for this

preprint (which was not certified by peer review) is the author/funder, who has granted bioRxiv a license to display the preprint in perpetuity. It is made available under aCC-BY-NC 4.0 International license.

1

\section{Abstract}

2 Childhood cancer is a major cause of child death in developed countries. Genetic interactions

3 between mutated genes play an important role in cancer development. They can be detected by

4 searching for pairs of mutated genes that co-occur more (or less) often than expected. Co-

5 occurrence suggests a cooperative role in cancer development, while mutual exclusivity points to synthetic lethality, a phenomenon of interest in cancer treatment research. Little is known about genetic interactions in childhood cancer. We apply a statistical pipeline to detect genetic interactions in a combined dataset comprising over 2,500 tumors from 23 cancer types. The resulting genetic

9 interaction map of childhood cancers comprises 15 co-occurring and 27 mutually exclusive

10 candidates. The biological mechanisms underlying most candidates are either tumor subtype,

11 pathway epistasis or cooperation while synthetic lethality plays a much smaller role. Thus, other

12 explanations beyond synthetic lethality should be considered when interpreting results of genetic 
1

\section{Introduction}

2 Cancer is the leading cause of disease-related child death in developed countries, despite increased

3

4 survival rates from $10 \%$ to over $80 \%$ in the last decades ${ }^{1}$. Moreover, survival rates differ greatly between different pediatric cancer types and many survivors suffer from severe side effects later on in life ${ }^{2,3}$. Therefore, it is of great importance to gain a better understanding of pediatric cancers and their potential treatments. Pediatric cancers are rare compared to adult cancers and are also fundamentally different. Insights gained from adult cancer studies are thus only for a small part applicable to childhood cancer types. While adult cancers usually occur late in life as the result of a gradual accumulation of somatic mutations, pediatric cancers are rather thought of as a developmental disease. Consequently, they usually have a different cell of origin compared to adult tumors ${ }^{4}$. In addition, they are thought to require a lower number of driver mutations for tumorigenesis and usually exhibit a much lower number of passenger mutations ${ }^{4}$. The exhaustive characterization of genetic alterations has only recently begun in pediatric cancers using wholegenome and exome sequencing approaches ${ }^{5}$. Larger numbers of sequenced tumors for pediatric cancers are now available, allowing the identification of genes that are frequently mutated across cancer genomes ${ }^{6,7}$.

Like many other diseases, most cancers do not arise from alterations in individual genes alone, but are the result of widespread genetic interactions between them ${ }^{8}$. Genetic interactions occur when the effect of combining two or more alterations in the genome cannot be predicted by adding up the effects of the individual alterations. Genetic interactions are known to be pervasive in model organisms $^{9-11}$. Efforts have also been initiated to map genetic interactions in adult cancer cells $\mathrm{s}^{12-15}$, but have so far been limited in pediatric cancer. One of the primary goals of such studies is to detect synthetic lethal relationships. Synthetic lethality is a type of genetic interaction in which simultaneous disruption of two genes results in cell death ${ }^{16}$ while the alteration of only one of the two genes yields a viable cell. For example, cancer cells that harbor mutations in BRCA1 or BRCA2 
are highly dependent on the function of $P A R P 1^{17}$. Exploiting these types of genetic interactions for therapeutic purposes can therefore advance precision medicine strategies to develop better, patient-tailored, cancer treatments ${ }^{18,19}$.

Multiple strategies can be employed to detect synthetic lethal and other genetic interactions in cancer cells (reviewed in ${ }^{20}$ ). For example, synthetic lethal gene pairs that are identified with highthroughput screens in model organisms such as yeast might indicate synthetic lethality between their human homologs ${ }^{12}$. Another approach is to use RNAi or CRISPR-Cas9 to perform knock-out experiments in human cancer cell lines ${ }^{13,14}$. However, candidate genetic interactions identified with these methods are often hard to validate, as they are context dependent and might not replicate in a different genomic background, cell type or cell environment ${ }^{19}$.

Another strategy to detect genetic interactions is to perform in silico statistical analyses in large collections of tumor genomes. Applying this approach to adult cancers has led to the identification of co-occurring and mutually exclusive pairs of alterations $\mathrm{s}^{21-24}$. A pair is co-occurring (or mutually exclusive) when two alterations co-occur more (or less) often than expected by chance. Mutually exclusive altered gene pairs can point to possible synthetic lethal candidates; mutated gene pairs that co-occur often probably co-operate to give the tumor cell a selective advantage. Numerous tools have been developed to identify co-occurring and mutually exclusive interactions between mutated genes in cancer (reviewed in ${ }^{25}$ ) and differ in the choice of underlying model, statistical approach, and incorporation of existing biological knowledge. However, the interpretation of mutual exclusivity and co-occurrence patterns is an essential but not straightforward step and in silico genetic interaction studies often lack a careful investigation of the biological mechanisms that can explain their results ${ }^{25-27}$.

Here we draw a comprehensive map of genetic interactions in pediatric cancer. We present a robust pipeline that implements two statistical tests to detect with high confidence significantly cooccurring and mutually exclusive gene pairs. We apply the pipeline on two pediatric cancer data 
sets, together consisting of over 2,500 tumors and 23 cancer types. We perform our analyses both per cancer type and at a pan-cancer level, while including the complete set of mutated genes and not restricting to driver genes only. We identify multiple co-occurring and mutually exclusive candidate gene pairs in both data sets. We next investigate potential underlying mechanisms that gave rise to patterns of co-occurrence and mutual exclusivity and provide estimates of their contribution in our result set. Our findings show that tumor subtype, pathway epistasis and cooperation are the main biological mechanisms underlying the results, as each explain over a quarter of the candidate genetic interactions found. Synthetic lethality plays a much smaller role, as

9 it explains only $10 \%$ of the candidate interactions. Taken this outcome we conclude that other 10 explanations beyond synthetic lethality should be considered when interpreting the results of 11 genetic interaction tests. 


\section{A combined genomic data set covering common pediatric cancers}

3

4

5

6

7

8

To systematically detect genetic interactions in pediatric cancer, a collection of tumor samples from two recently published pediatric cancer data sets was used as a starting point ${ }^{6,7}$. The first data set ${ }^{6}$, hereafter called DKFZ, consists of 961 tumors from 23 distinct cancer types (Figure 1a). The second data set ${ }^{7}$, hereafter called TARGET, contains 1,699 tumors from six distinct cancer types (Figure 1b). The combined cohort covers major childhood cancer types including central nervous system tumors, hematological tumors as well as solid tumors. While the DKFZ data set has a focus on central nervous system tumors, the TARGET data set has an emphasis on hematological tumors, thus complementing each other in the extent to which individual tumor types are covered. The data sets contain whole-genome sequenced (WGS) and whole-exome sequenced (WES) tumor samples. Only primary tumors were used for detecting genetic interactions.

Genetic alterations that were considered for the genetic interaction tests include single nucleotide variants (SNVs) as well as small insertions and deletions (indels). Only SNVs and indels in coding regions that likely have a functional effect were used for further analyses. All genes that harbor one or more mutations were identified, not limiting the analysis to just the candidate driver genes that are frequently mutated across samples, earlier identified as significantly mutated genes (SMGs). The number of mutated genes per individual tumor varies greatly across different pediatric cancer types (Figure $1 c, d$ ) as was also reported before ${ }^{6,7}$. Hepatoblastoma (HB) has the lowest number of mutated genes (median $=1$ ), whereas Burkitt's lymphoma $(B L)$ has the highest number of mutated genes (median $=28$ ). Several individual tumor samples have no detected mutations of the types considered here. To avoid false positives, seven DKFZ samples and two TARGET samples with an extremely high number of mutated genes (denoted as hypermutators) were excluded from further downstream analyses. After filtering, a total of 2,461 tumors remained in the combined cohort (829 
bioRxiv preprint doi: https://doi.org/10.1101/2020.11.17.385120; this version posted December 5,2020 . The copyright holder for this preprint (which was not certified by peer review) is the author/funder, who has granted bioRxiv a license to display the preprint in perpetuity. It is made available under aCC-BY-NC 4.0 International license.

tumors from DKFZ; 1,632 tumors from TARGET) and these were used for investigating genetic interactions (See Methods and Supplemental Table S1 for more details about the filtering steps).

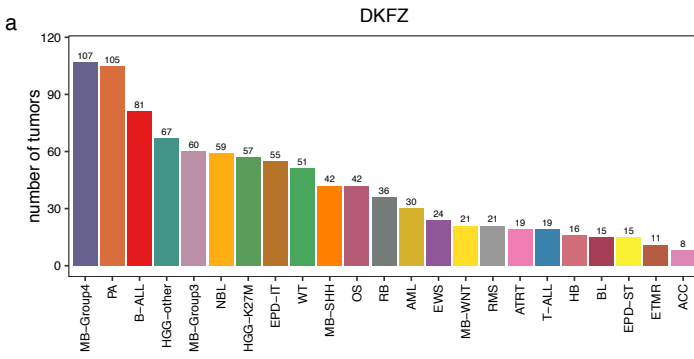

c

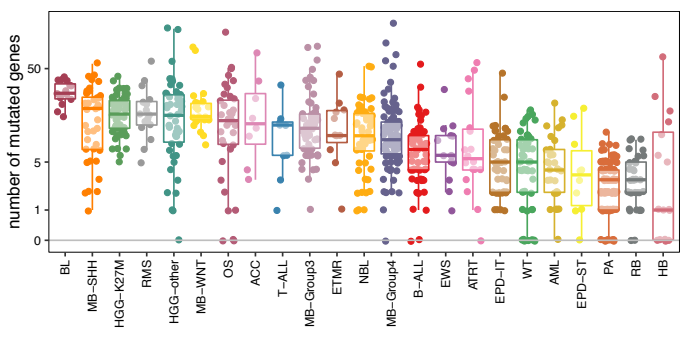

b
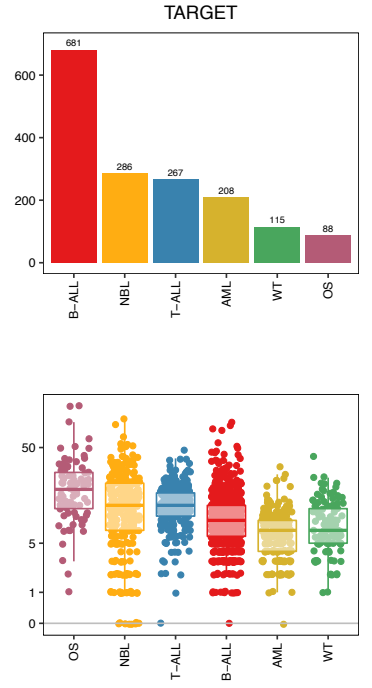

ACC (adrenocortical carcinoma)

AML (acute myeloid leukemia)

ATRT (atypical teratoid/rhabdoid tumors)

B-ALL (B-cell acute lymphoblastic leukem

BL (Burkitt's lymphoma)

EPD-IT (infratentorial ependymoma)

EPD-ST (supratentorial ependymoma)

ETMR (embryonal tumors with multilayered rosettes)

EWS (Ewing's sarcoma)

HB (Hepatoblastoma)

HGG-K27M (high-grade glioma - K27M)

HGG-or (nigh-gade glona-

MB-Group3 (medulloblastoma - Group3)

B-Group4 (medulloblastoma - Group4)

MB-SHH (medulloblastoma - SHH)

MB-WNT (medulloblastoma - WNT)

NBL (neuroblastoma)

(

PA (pilocytic astrocytoma)

RB (retinoblastoma)

T-ALL (T-cell acute Iymphoblastic leukemia)

WT (Wilm's tumor)
3

Figure 1. Number of samples and mutated genes per cancer type. $(a, b)$ The number of samples per cancer type in the DKFZ (a) and TARGET (b) data sets used in this study before sample filtering (e.g. removal of hypermutators, relapse tumors). (c, d) Number of mutated genes identified in each individual tumor per cancer type for the DKFZ (c) and TARGET (d) data sets after sample filtering. Points on the horizontal axis represent tumor samples without any mutated genes. Mutated genes are defined as genes that harbor at least one exonic mutation with a likely functional consequence. Only SNVs and small indels were considered.

\section{A robust statistical pipeline to detect high confidence genetic interactions}

To detect potential genetic interactions that play a role in pediatric cancer, we developed a

statistical pipeline to detect pairs of altered genes that co-occur significantly more (or less) often

than expected given their individual frequencies (Figure 2, Methods). A pan-cancer analysis as well as a test per cancer type to detect cancer-type specific interactions on either the DKFZ or TARGET

data set were performed. As each data set was produced with its own technical and filtering procedures, we applied the tests on each data set separately as merging both sets would possibly lead to false positives. The resulting candidate gene pairs can point to possible genetic interactions between genes that play an important role in pediatric cancer. 
1

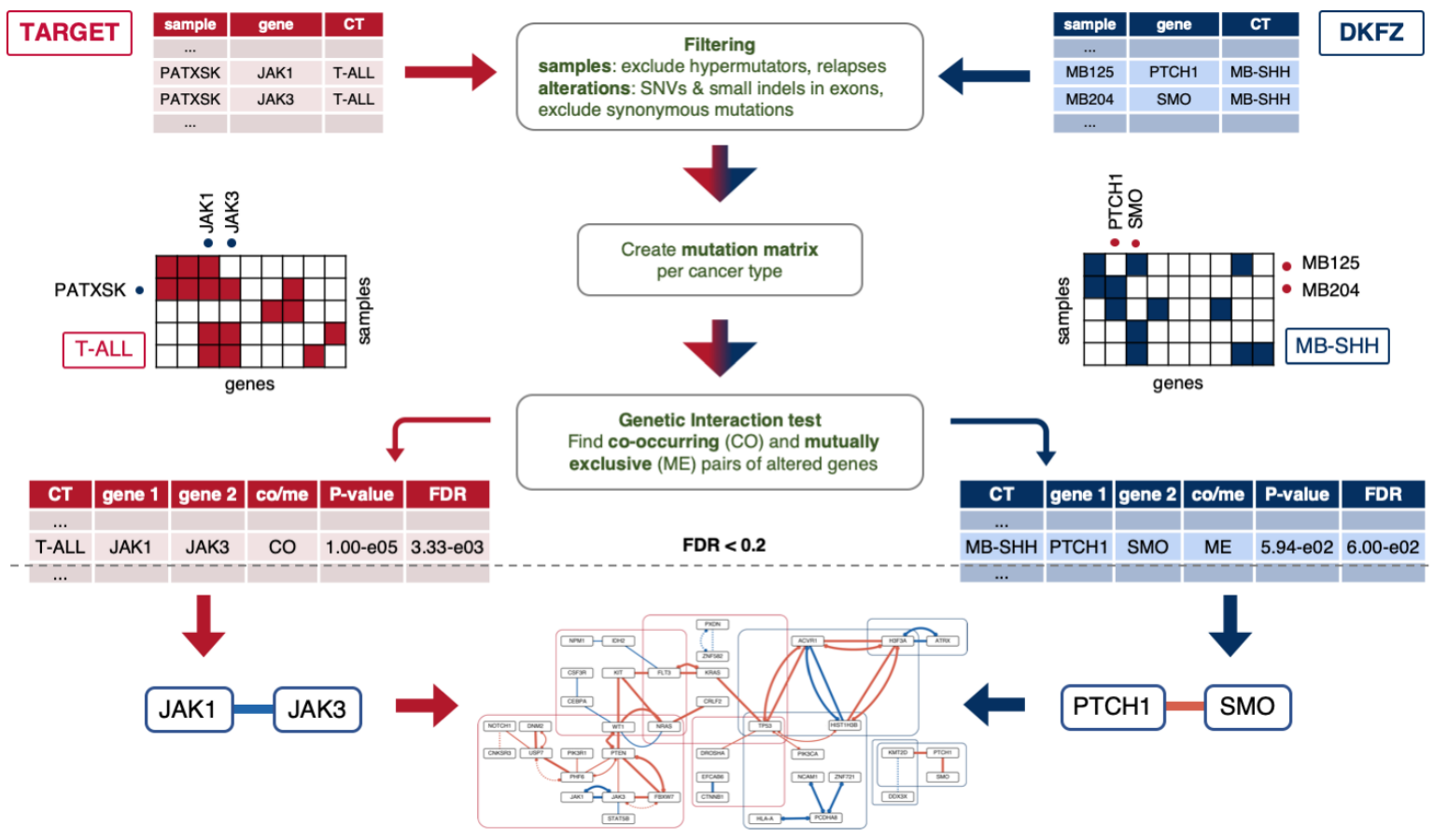

Figure 2. Workflow depicting the statistical pipeline to detect genetic interactions. Starting with the original mutation files from the TARGET and DKFZ data set, samples and alterations are first filtered after which a mutation matrix is created to record which samples contain mutations in a certain gene. Here, the candidate gene pairs JAK1-JAK3 and PTCH1-SMO are shown as example. The matrix serves as input for the Permutation and WeSME test to detect significantly co-occurring or mutually exclusive pairs of altered genes. The resulting candidates from both data sets are merged into a genetic interaction map of childhood cancer.

In short, the approach is based on two previously published tests for genetic interactions in cancer.

The first test, which we will call hereafter the Permutation test, was described earlier ${ }^{23}$ and

successfully identified various genetic interaction candidates in adult cancers. It starts with the creation of a binary mutation matrix listing which genes are mutated in which tumor samples. Next, for each gene pair, the number of samples in which both genes are mutated are counted (cooccurrence count). Extreme low values point to mutually exclusive candidate gene pairs, while high counts suggest co-occurring interactions. To infer the significance of such extreme counts, a null distribution is created by repeatedly permuting the mutation matrix while keeping its margins fixed. In each permuted matrix and each gene pair co-occurrence is again counted and co-occurrence $P$ values $\left(P_{\text {co }}\right)$ are estimated as the proportion of 'permuted' counts equal or higher than the observed 
count. $P$-values for mutual exclusivity $\left(P_{\mathrm{me}}\right)$ are inferred in a similar way by taking the proportion equal or lower than the observed count.

The second genetic interaction test, named Weighted Sampling based Mutual Exclusivity or WeSME in short, is based on the same principle as the Permutation test, using a mutation matrix, but has implemented several improvements that significantly speeds up the testing procedure, while producing highly similar results ${ }^{28}$. Most importantly, to assign co-occurrence and mutual exclusivity $P$-values to gene pairs, a null distribution of counts is created, not by permuting the mutation matrix, but by sampling patients with a probability weighted by their mutation rate.

$P$-values resulting from both the Permutation test as well as the WeSME method were corrected for multiple testing by estimating an empirical false discovery rate (FDR). To estimate the FDR, a null distribution of $P$-values was obtained by testing a random sample of permuted matrices. Gene pairs scoring an FDR $<0.2$ and with $P$-values $<0.1$ were considered candidate genetic interactions. To produce high confidence results each WeSME test was repeated ten times (using different randomization seeds) and only those pairs that scored significantly in at least nine out of ten tests were considered as high confidence pairs.

\section{A comprehensive map of potential genetic interactions in pediatric cancer}

Applying the Permutation and WeSME test on each cancer type in the TARGET data set resulted in a total of 28 candidates divided over the three leukemia cancer types T-ALL (14), AML (8), B-ALL (4) and Wilms Tumor (2) (Table 1 and Figure 3). In the pan-cancer test we detected eleven candidates, ten of which were also discovered in the test per cancer type. Of all 29 unique candidate gene pairs, nine were co-occurring, and 20 were mutually exclusive. Of all candidates, eighteen were detected in both the Permutation as well as the WeSME test, sixteen in WeSME only and five in the Permutation test only. Seventeen candidate interactions were discovered earlier in the same data set $^{7}$, and twelve candidates are new findings in this study. In the DKFZ cancer types we found a total 
bioRxiv preprint doi: https://doi.org/10.1101/2020.11.17.385120; this version posted December 5,2020 . The copyright holder for this preprint (which was not certified by peer review) is the author/funder, who has granted bioRxiv a license to display the preprint in perpetuity. It is made available under aCC-BY-NC 4.0 International license.

of eight candidates, all in High Grade Glioma (HGG) and Medulloblastoma (MB) sub types, namely HGG-K27M (4), HGG-other (1), MB-SHH (2), and MB-WNT (1) (Table 1 and Figure 3). Five of these candidates were also found in the pan-cancer analysis, together with five pan-cancer only candidates. In total we detected six co-occurring and seven mutually exclusive candidates. Of all candidates, fifteen scored significant in both the Permutation and WeSME test, two in WeSME only and one exclusively in the Permutation test. Only two candidates (ACVR1-HIST1H3B and ATRX$\mathrm{H} 3 \mathrm{~F} 3 \mathrm{~A}$ ) were detected as potential genetic interactions previously in the same data set ${ }^{6}$. Combining the results from both data sets leads to 42 candidate genetic interactions, of which 27 mutually exclusive and 15 co-occurring interactions (see Supplemental Table S2 for more details).

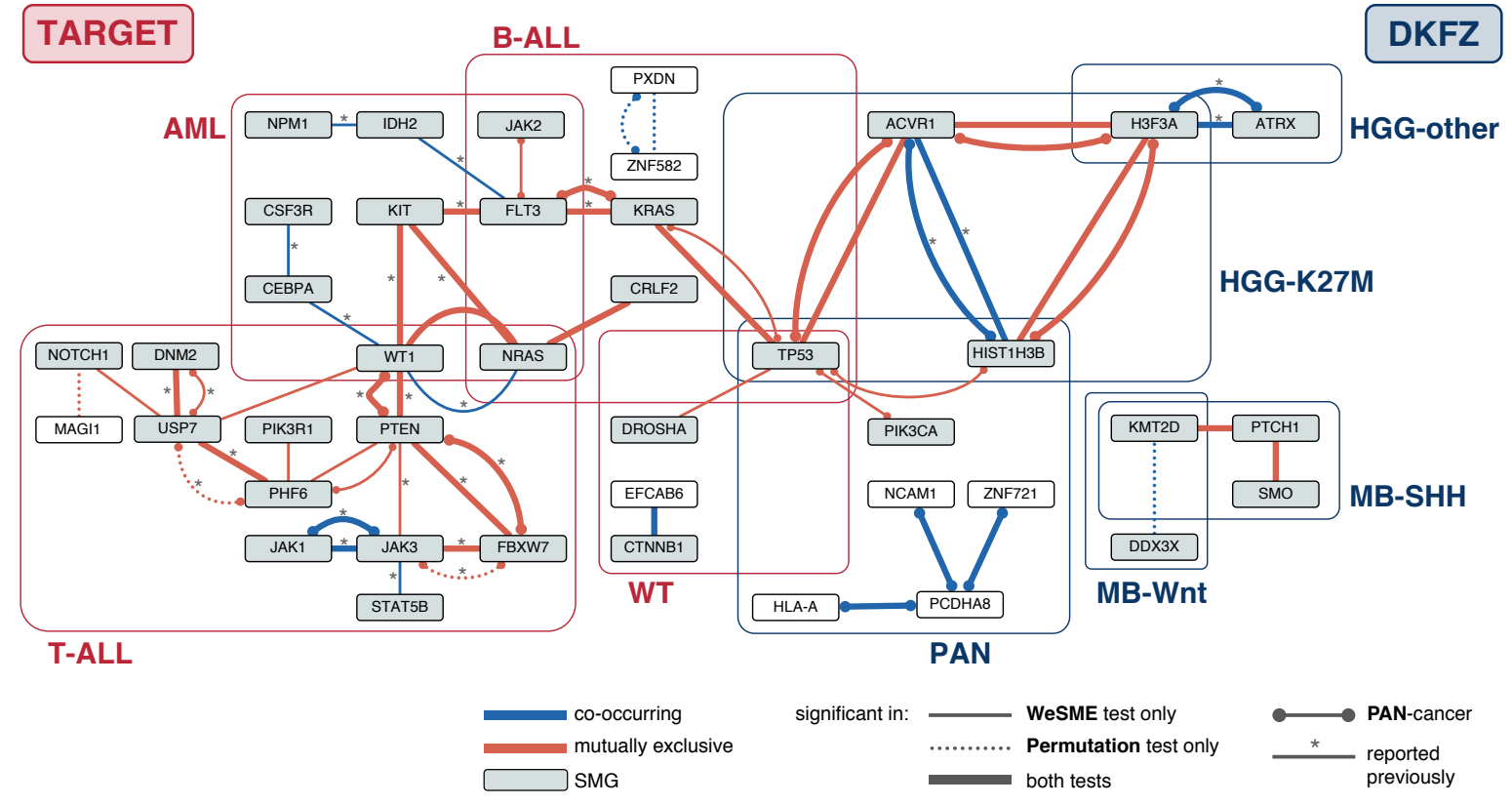

Figure 3. Candidate gene pairs resulting from the Genetic Interaction detection pipeline. Graph depicting candidate genetic interactions found in either TARGET (red boxes) or DKFZ (blue boxes). Mutually exclusive pairs are indicated in red, co-occurring pairs in blue. SMG: significantly mutated genes. 'reported previously' refers to gene pairs that were detected as significantly co-occurring or mutually exclusive in the studies accompanying the TARGET $T^{7}$ and $D K F Z^{6}$ data sets. 
2 Most genetic interaction candidates that were detected involve possible cancer driver genes, namely genes that were determined to be significantly mutated genes (SMGs) in previous studies ${ }^{6,7}$.

4 However, in six interactions discovered in this study, non-SMGs were involved, and they are

5 relatively often co-occurring (five candidates) and pan-cancer specific (three candidates). This

6 suggests that the inclusion of the whole set of genes instead of the set of SMGs, can lead to novel

7 discoveries.

Table 1. Number of candidate genetic interactions in both data sets.

\section{TARGET DKFZ}

\begin{tabular}{lrr}
\hline Number of candidates & 39 & 18 \\
All & 29 & 13 \\
Unique & 9 & 6 \\
\hline Type of interaction & 20 & 7 \\
$\quad$ Co-occurring & & \\
Mutually exclusive & 28 & 8 \\
\hline Which analysis & 11 & 10 \\
$\quad$ Per cancer type & $(10)$ & $(5)$ \\
$\quad$ PAN & 17 & 2 \\
$\quad$ (overlap cancer type) & 12 & 11 \\
\hline Previously reported & \\
$\quad$ In original paper & 18 & 15 \\
Novel candidate & 16 & 2 \\
Significant in which test & 5 & 1 \\
Both tests & \\
WeSME only & \\
$\quad$ Permutation test only & \\
&
\end{tabular}

13 We find about two times more mutually exclusive than co-occurring candidate interactions with our

14 pipeline. The most likely explanation is a technical one. If two mutated genes have a cooperative

15 relationship, but each have a low mutation frequency, the chance of occurring together in the same 
tumor is even lower and one needs large numbers of samples to have enough power to discover such co-occurring relationships.

The fact that we find less candidate genetic interactions than reported in the earlier two studies on the same data sets has a technical ground as well. Our approach differs in several aspects from the tests applied in the previous studies. First, we include all mutated genes instead of focusing on SMGs only. This way we will detect genetic interactions that involve non-driver genes, but we also introduce more noise which decreases the power of the test. Furthermore, as our pipeline is based on randomizations per cancer type, instead of using e.g. a Fisher Exact test, we control for the underlying data structure and are more likely to avoid false positives. Third, we applied thresholds on the minimum number of co-occurrences to exclude low-confidence results. Overall, this results in a more conservative estimate of the number of candidate pairs that we predict compared to the previous studies.

Interestingly, none of the discovered gene pairs were found in more than one cancer type albeit several candidates were confirmed in the pan-cancer analysis. Moreover, none of the candidates were detected in the other data set. Even the genes involved in significant gene-pairs appear to be data set specific as only TP53 is part of candidate interactions in both the TARGET and DKFZ data set.

\section{Sample size explains lack of overlap in candidates for cancer types present in both data sets}

Even though the six cancer types of the TARGET data set are also present in the DKFZ data set, none of the candidates found in the TARGET data set were confirmed in the DKFZ analysis. The most probable explanation would be the fact that the number of samples of these cancer types is much lower in the DKFZ set compared to the TARGET set. Indeed, one of the important requirements to detect genetic interactions in silico, is to test large numbers of samples to have sufficient statistical power. The number of samples is usually not a limiting factor for adult cancers, but sample size can be an issue in rare diseases such as pediatric cancers. 
bioRxiv preprint doi: https://doi.org/10.1101/2020.11.17.385120; this version posted December 5,2020 . The copyright holder for this preprint (which was not certified by peer review) is the author/funder, who has granted bioRxiv a license to display the preprint in perpetuity. It is made available under aCC-BY-NC 4.0 International license.

1 To investigate if sample size indeed is the underlying cause, we performed a down-sampling analysis where we performed the WeSME test on random subsamples of the four cancer types for which we detected candidates in the TARGET data set, namely B-ALL, T-ALL, AML and WT. As shown in Figure 4, we observe a wide variation in the number of candidates, especially when testing larger sample sizes (200-400). Repeating the WeSME test ten times and only consider candidates that score significantly in at least nine out of ten tests thus proves to be a good strategy to detect highconfidence candidates. More importantly, the number of discoveries drops dramatically with sample sizes of 100 and below, which explains the lack of candidates in these four cancer types in the DKFZ data set, as their number of samples lies in the range between 11 and 51.

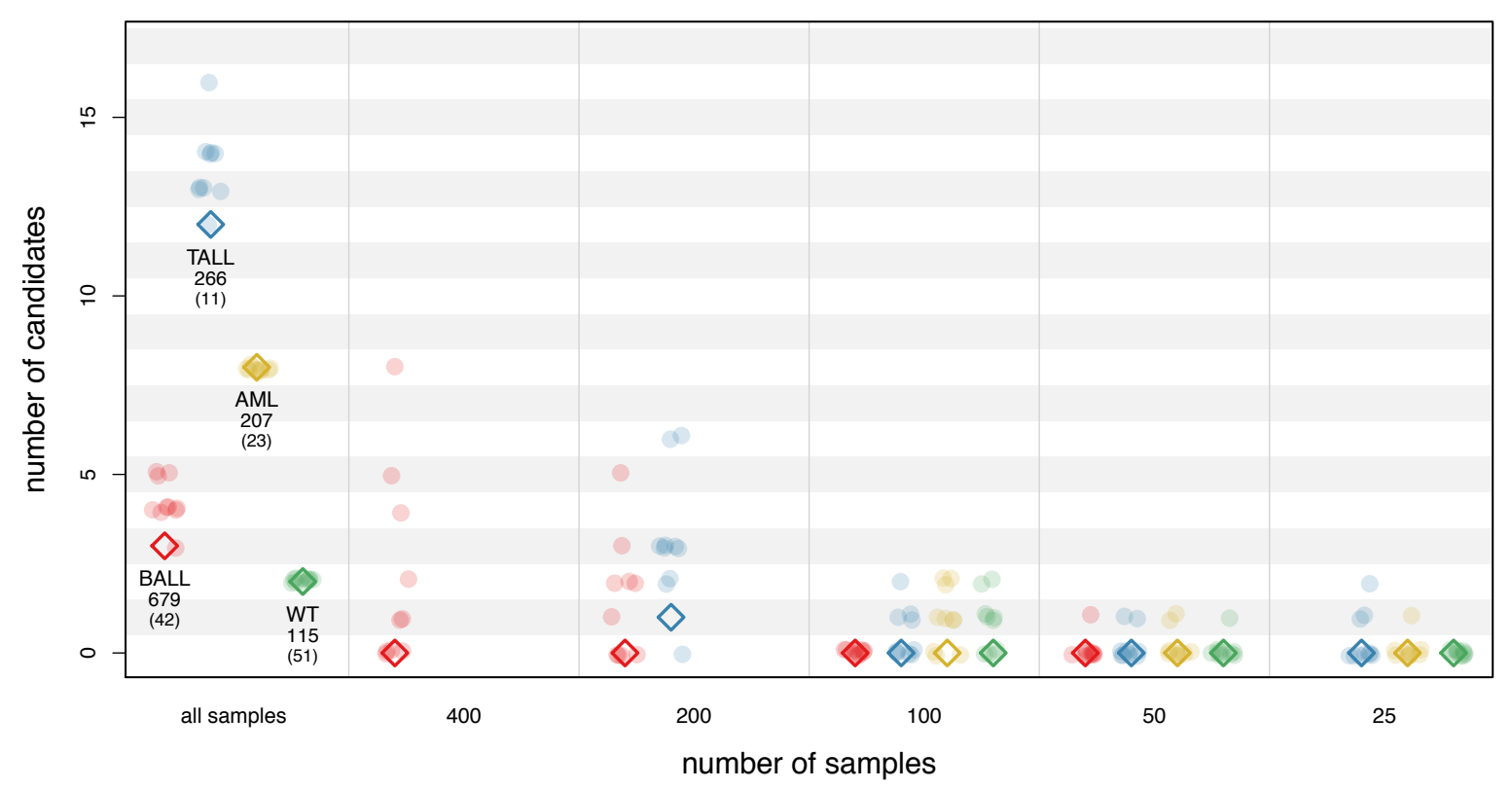

Figure 4. Down-sampling the TARGET data set greatly reduces number of candidates. Number of candidate pairs for the WeSME test on TARGET cancer types B-ALL, T-ALL, AML and WT using either all samples, or downsampled data sets. Dots represent the number of candidates for each of the ten replicates per sample size. Diamonds indicate the number of high confident candidates (being a significant candidate in at least nine out of ten tests). The first category (all samples) shows for each cancer type the original number of samples in the TARGET and -in parentheses- in the DKFZ data set. 
2 Recently it has been shown by Van de Haar and colleagues that tumor subtype and tumor mutation load can be confounding factors in tests to detect genetic interactions in cancer data sets ${ }^{27}$. When

4

the mutation frequency of a gene is strongly associated with the tumor mutation load of a sample, such a gene will likely turn up as a mutually exclusive candidate with mutated genes that have a negative association with tumor mutation load. We investigated whether tumor mutation load can explain part of our candidate genetic interaction pairs. We calculated the tumor mutation load per sample and the mutation load association $\left(M L A^{27}\right)$ of each candidate gene, where MLA is an indication of the association between the mutation load and likelihood of being mutated (Methods). Compared to the Van de Haar study, which found MLA scores between minus four and ten with a median of five, we find in general much lower MLA values per gene (Supplemental Figures S1 and S2), indicating that overall there is no strong correlation with tumor mutation load in pediatric cancer. This most likely can be explained by the much smaller number of passenger mutations in pediatric cancers.

Next, we defined as 'suspect' all candidate mutually exclusive gene pairs with an MLA difference larger than three and at least one gene having an MLA larger than three or with both genes having an MLA larger than three in case of co-occurrence. We found seven of such suspect candidates, all belonging to T-ALL (Figure 5, Supplemental Table S3, Supplemental Figure S3). Most T-ALL tumors can be assigned to different subtypes, characterized by mutually exclusive structural aberrations and subtype specific expression profiles ${ }^{29-31}$. More specifically, T-ALL subtypes reflect the maturation state of the T-cell progenitor from which the tumor developed. Since we do have subtype annotation available for almost all available T-ALL samples from the TARGET data set (Methods), we examined whether there is an association between tumor subtype and tumor mutation load. Indeed, there is a clear difference between subtypes, with a tendency of tumor types associated with primitive T-cell progenitors carrying more mutations than subtypes associated with more differentiated precursors (Figure 5). Four of the seven suspect mutually exclusive candidate T-ALL 
bioRxiv preprint doi: https://doi.org/10.1101/2020.11.17.385120; this version posted December 5,2020 . The copyright holder for this

preprint (which was not certified by peer review) is the author/funder, who has granted bioRxiv a license to display the preprint in perpetuity. It is made available under aCC-BY-NC 4.0 International license.

1

2

3

4

gene pairs can likely be explained by the fact that mutated genes in these pairs are enriched in

different subtypes with different mutation loads (Supplemental Table S3). The remaining three gene

pairs involve one of the commonly mutated genes NOTCH1 and FBXW7 and could be false positives

(Supplemental Figure S3), but are likely the result of other biological mechanisms.

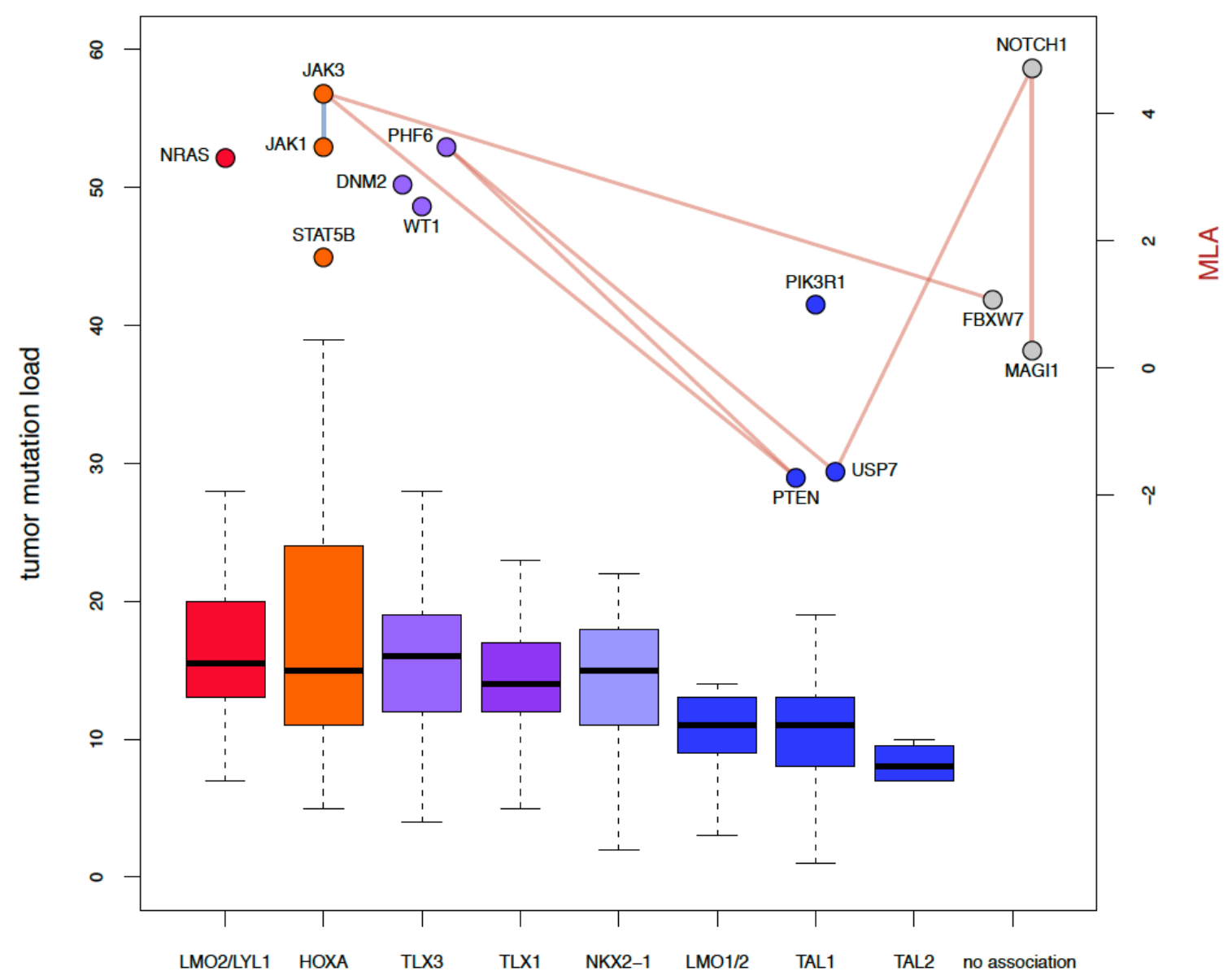

5

Figure 5. Tumor mutation load is lower in more mature T-ALL subtypes. Sample tumor mutation load for each T-all subtype, with subtypes ordered (left to right) from early to late T-cell progenitor stage. Genes that were part of a candidate genetic interaction and that were significantly associated with a T-ALL subtype are depicted in the figure with their corresponding subtype and their MLA score (secondary vertical axis). Subtype ordering, coloring and assignment to genes were reproduced from Figure 3 in ${ }^{31}$. Edges between genes represent 'suspect' candidate genetic interactions found in T-ALL, defined as mutually exclusive gene pairs (shown in red) with one gene having an MLA score $>3$ and together having a difference in MLA score $>3$ or a co-occurring pair (shown in blue) with both genes having an MLA > 3 . 


\section{Four biological mechanisms underly most candidate gene pairs}

Next, we investigated for each of the candidate genetic interactions which underlying biological mechanism could explain their mutually exclusive or co-occurring relationship. This entailed consulting experts in the field of the corresponding cancer types as well as an extensive literature search. We considered the function of the individual genes, known relationships between the found gene pairs and their role in cancer, with specific focus on the cancer type in which the candidate was detected. In addition, we developed a Candidate Reporting Tool, which is an R shiny web application (available as of December 1, 2020 on http://gi-analysis.bioinf.prinsesmaximacentrum.nl/) to aid in the inspection of mutational information on each candidate gene pair, producing multiple visualizations together with a table listing the biological or pathogenic consequence of each individual mutation in the corresponding genes. We have listed all candidates in Table 2 together with the most likely biological mechanism that can explain the detected mutual exclusivity or cooccurrence.

As indicated before, many of the candidate gene pairs have a co-occurring or mutually exclusive relationship because the cancer type in which they were detected actually consists of multiple sub types. Apart from the T-all examples described above, this is for example the case for the high-grade glioma K27M group. The mutually exclusive candidate gene-pair HIST1H3B-H3F3A in this group can be explained by the fact that patients with this particular cancer type can be divided into two subgroups, defined by K27M mutations in either the HIST1H3B or H3F3A genes that code for histones H3.1 and H3.3 respectively ${ }^{32-34}$. Typically, patients in the H3.1 subgroup also carry ACVR1 mutations, explaining the ACVR1-HIST1H3B co-occurrence and ACVR1-H3F3A mutual exclusivity relationships. Similarly, TP53 is almost exclusively mutated in the H3.3 group, again explaining mutually exclusivity between TP53 and ACVR1. All these candidates, together with the mutually 
exclusive TP53-HIST1H3B pair, were also found in the PAN cancer test, due to this strong signal in HGG-K27M, rather than similar patterns in other cancer types.

Other candidate gene pairs can be explained by pathway epistasis, the phenomenon in which the

4 mutation of one gene will have the same effect on the activation or inactivation of a pathway as mutating another (downstream) gene. In such cases, mutating either genes will result in the same effect, and therefore one mutation is enough for the tumor cell to gain a selective advantage. For example, mutations in PTCH1 and SMO were found to be mutually exclusive in the Medulloblastoma SHH cancer type. Both genes are part of the Sonic Hedgehog (Shh) pathway, which is assumed to have a tumor driver role in this type of medulloblastoma ${ }^{35}$. PTCH1 normally inhibits activation of SMO, but the presence of Hedgehog (Shh) leads to suppression of PTCH1 and consecutive activation of the Shh pathway by SMO. It has been shown that SMO inhibitors are most effective in MB-SHH patients with $S M O$ or $P T C H 1$ mutations ${ }^{36}$, suggesting that $S M O$ mutations found in MB-SHH samples do not have a knock-out effect on the gene, but more likely release or circumvent its inhibition by PTCH1. This might explain the mutually exclusive relationship found between altered PTCH1 and SMO genes, as both have the same effect and therefore are redundant mutations.

Mutual exclusivity could also point to a possible synthetic lethal relationship between genes, where mutating either of the two genes has no effect on cell viability but mutating both genes will result in cell death. We found several candidate interactions that could be driven by synthetic lethality, although no previous studies were found that confirm such a relationship. One such gene pair is NRAS-WT1, found to be mutually exclusive in AML. NRAS is, together with KRAS, part of the GTPase family of genes which are often activated in cancer. It has been shown that in KRAS-dependent tumors, inactivation of WT1 will reduce tumor formation ${ }^{37}$. If this effect also applies to NRAS mutated tumors, NRAS-WT1 could be a synthetically lethal gene pair. RAS genes act downstream of KIT in the RTK signaling pathway, therefore the synthetically lethal relationship between KRAS/NRAS and WT1 might also translate to the mutually exclusive KIT-WT1 candidate in AML. Interestingly, the 
gene-pair NRAS-WT1 is a co-occurring candidate in T-ALL. WT1 is known to play different roles in cancer, acting both as tumor suppressor and oncogene, where its function often depends on which isoform is expressed ${ }^{38}$. The apparent adverse interaction between NRAS and WT1 in AML and T-ALL might be due to these different roles of WT1 in cancer development and warrants further investigation.

6 Candidate co-occurring gene pairs possibly point to a cooperative relationship, in which the

7 mutation in one gene reinforces the effect of mutating the other gene. An example of such gene pair

8 is ATRX-H3F3A in the HGG Other group. The H3F3A mutations in this group are not K27M, but

$9 \mathrm{G} 34 \mathrm{R} / \mathrm{V}$ H3 mutations, another recurrent H3F3A alteration in high grade gliomas. ATRX regulates chromatin remodeling and transcription and plays an important role in maintaining genome stability by recruiting $\mathrm{H} 3.3$ at telomeres and pericentric heterochromatin. It has been suggested that the loss of $A T R X$ could prevent mutant $\mathrm{H} 3.3$ from altering transcription of specific oncogenes ${ }^{39}$, which would suggest a co-operative role of both altered genes in tumorigenesis.

Considering all candidate genetic interactions from both data sets, we clearly see that cancer subtype (12 gene pairs) is the most likely underlying cause in about $44 \%$ of the mutually exclusive candidates and $29 \%$ of all candidates (Table 2). Pathway epistasis (11 pairs) accounts for $41 \%$ of the mutually exclusive candidates ( $26 \%$ of all candidates) and $15 \%$ ( 4 pairs) may be caused by synthetic lethality ( $10 \%$ of all candidates). About $73 \%$ of co-occurrences (11 pairs, $26 \%$ of all candidates) could be attributed to co-operation while the remaining $27 \%$ (4 pairs, $10 \%$ of all candidates) are likely false positives. 
bioRxiv preprint doi: https://doi.org/10.1101/2020.11.17.385120; this version posted December 5,2020 . The copyright holder for this

preprint (which was not certified by peer review) is the author/funder, who has granted bioRxiv a license to display the preprint in perpetuity. It is made available under aCC-BY-NC 4.0 International license.

1 Table 2. Candidate genetic interactions and their underlying mechanisms

DKFZ

\begin{tabular}{llllll}
\hline cancer type & gene1 & gene2 & co/me & most likely mechanism & reference(s) \\
\hline HGG-K27M & ACVR1 & HIST1H3B & co ${ }^{*}$ & cooperation & 40 \\
& & & & & 32,33 : subtype \\
& ACVR1 & H3F3A & me $^{*}$ & subtype & 32,33 \\
& ACVR1 & TP53 & me $^{*}$ & & \\
& H3F3A & HIST1H3B & me $^{*}$ & & $39,41,42$ \\
\hline HGG-other & ATRX & H3F3A & co $^{*}$ & cooperation & 43,44 \\
\hline MB-SHH & KMT2D & PTCH1 & me & pathway epistasis & 36 \\
\hline MB-WNT & DTCH1 & SMO & me & pathway epistasis & 45 \\
\hline PAN & HLA-A & KMT2D & co & cooperation & \\
& NCAM1 & PCDHA8 & co & false positives & \\
& PCDHA8 & ZNF721 & co & & 32,33 \\
& HIST1H3B & TP53 & me & subtype & $46:$ cooperation TP53-H3.3 \\
& & & & & 47 \\
\hline
\end{tabular}

\section{TARGET}

\begin{tabular}{|c|c|c|c|c|c|}
\hline cancer type & gene1 & gene2 & $\mathrm{co} / \mathrm{me}$ & most likely mechanism & reference(s) \\
\hline \multirow[t]{8}{*}{$\mathrm{AML}$} & CEBPA & CSF3R & co & cooperation & 48,49 \\
\hline & CEBPA & WT1 & co & cooperation & 50,51 \\
\hline & FLT3 & IDH2 & co & cooperation & $52-54$ \\
\hline & IDH2 & NPM1 & co & cooperation & 53 \\
\hline & FLT3 & KIT & me & pathway epistasis & 55 \\
\hline & KIT & NRAS & me & pathway epistasis & \\
\hline & KIT & WT1 & me & synth lethality & 37 \\
\hline & NRAS & WT1 & me & synth lethality & 37,56 \\
\hline \multirow[t]{4}{*}{ B-ALL } & PXDN & ZNF582 & $\mathrm{CO}^{*}$ & false positive & \\
\hline & CRLF2 & NRAS & me & subtype & 57 \\
\hline & FLT3 & KRAS & $\mathrm{me}^{*}$ & pathway epistasis & $55,58,59$ \\
\hline & KRAS & TP53 & $\mathrm{me}^{*}$ & subtype & 57 \\
\hline \multirow[t]{14}{*}{ T-ALL } & JAK1 & JAK3 & $\mathrm{CO}^{*}$ & cooperation & 60 \\
\hline & JAK3 & STAT5B & co & cooperation & 61 \\
\hline & NRAS & WT1 & co & cooperation & \\
\hline & DNM2 & USP7 & $\mathrm{me}^{*}$ & subtype & 31 \\
\hline & FBXW7 & JAK3 & $\mathrm{me}^{*}$ & pathway epistasis & $29,62,63$ \\
\hline & FBXW7 & PTEN & $\mathrm{me}^{*}$ & pathway epistasis & $29,62,63$ \\
\hline & JAK3 & PTEN & me & pathway epistasis & $30,64,65$ \\
\hline & MAGI1 & NOTCH1 & me & pathway epistasis & 66 \\
\hline & NOTCH1 & USP7 & me & synthetic lethality & 67 \\
\hline & PHF6 & PIK3R1 & me & subtype & 30 \\
\hline & PHF6 & PTEN & $\mathrm{me}^{*}$ & subtype & 30 \\
\hline & PHF6 & USP7 & $\mathrm{me}^{*}$ & subtype & 31 \\
\hline & PTEN & WT1 & $\mathrm{me}^{*}$ & synthetic lethality & $30,64,65$ \\
\hline & USP7 & WT1 & me & subtype & 31 \\
\hline \multirow[t]{2}{*}{ WT } & CTNNB1 & EFCAB6 & CO & cooperation & 68 \\
\hline & DROSHA & TP53 & me & subtype & 69 \\
\hline PAN & FLT3 & JAK2 & me & pathway epistasis & 70 \\
\hline
\end{tabular}


Here, we built a comprehensive map of genetic interactions in childhood cancer. We applied a robust statistical pipeline to detect mutually exclusive and co-occurring altered gene pairs in two pediatric pan cancer data sets. We implemented two related methods for genetic interaction detection: the permutation test and the WeSME test. Most of the 15 co-occurring and 27 mutually exclusive candidate gene pairs were detected with both tests, confirming that the faster WeSME test is a good alternative for the permutation test. Indeed, we find a strong correlation between the p-values of all tested gene pairs that were produced by both tests (Pearson's $r=0.97, p$-value $<2.2 \mathrm{e}-16$ ). Note however that the WeSME test has a slight tendency toward lower mutual exclusivity p-values, while the Permutation test yields lower co-occurrence p-values (Supplemental Figure S4) as was shown earlier by Kim et al. ${ }^{28}$. It has been previously shown that genetic interactions are tissue and cancer type specific ${ }^{23}$. Indeed, we confirm this finding in our current study as no candidate genetic interaction was detected in more than one cancer type. This is partly due to the fact that, apart from a few commonly mutated genes in various cancers, most cancer types are characterized by recurring alterations in specific genes, showing that the process of oncogenesis is unique within each tumor (sub) type. The sensitivity to certain genetic hits at a specific maturation state of the cell of origin is a main driver underlying this process. As an additional consequence, we have more power to find genetic interactions between these frequently mutated genes, compared to rarely mutated genes.

Although all cancer types in the TARGET data set are also part of the DKFZ set, we did not reproduce the TARGET candidates when testing the same cancer types in DKFZ. We showed with down sampling that the most likely explanation for this lack of overlap is the reduced samples size for these cancer types in DKFZ. This stresses the importance of having a large enough data set to detect genetic interactions. Especially in pediatric cancer this poses a challenge as most of the cancer types are rare and data sets produced within one institute are relatively small. Current initiatives to collaborate 
between institutes and countries to build large (pediatric) cancer resources consisting of combined genomic data sets is a key step towards a solution. Examples of such collaborations are the GENIE project $^{71}$, the Children's Brain Tumor Tissue Consortium ${ }^{72}$ and St. Jude Cloud ${ }^{73}$. However, merging data sets imposes new challenges, since the application of different protocols, sequencing techniques, and quality filtering methods will introduce confounding factors leading to batch effects and consequently false positives. Genetic interaction tests applied on merged data sets should therefore take the underlying heterogeneity of these different sources into account, for example by using a similar approach as we presented here for the PAN cancer test, where we count cooccurrences and mutual exclusivity over the whole set, but apply the randomizations within cancer types. This approach could be easily translated to a framework in which randomizations take place among samples from the same source, while summary statistics are collected over the combined data set.

For practical utility in mechanism-of-action based therapies as well as for understanding the biology driving cancer progression, we need a much better understanding of the mechanisms underlying genetic interactions. Mutual exclusivity found between altered gene pairs is often the result of mutated genes being specific for different cancer subtypes. For the same reason co-occurrence will be detected between mutated genes that are enriched in certain cancer subtypes. This underscores the importance to distinguish between subtypes in the clinic, because it is likely that they will respond differently to the same treatment. Co-occurrence could also indicate possible cooperation between both genes in cancer development. Finding such cooperating pairs will give us more insight in the pathways involved in tumorigenesis. Pathway epistasis is another source of mutual exclusivity, as mutating genes that are part of the same pathway may have the same downstream effect, and as a result having only one gene mutated is sufficient to have a beneficial effect for the tumor. Mutual exclusivity or co-occurrence could also occur as a result of MLA bias, leading to false positive candidates. Indeed, we found several mutually exclusive candidate gene pairs in T-ALL with large MLA differences and one co-occurring pair of high MLA genes. Most of these 'suspect' gene pairs are 
however more likely explained by the fact that the genes involved are associated with different subtypes. Interestingly, we also showed that in T-ALL mutation load decreases with maturation state of the tumor cell. Synthetic lethality is another explanation for mutual exclusivity. Synthetic lethality is of particular importance for cancer research because it can be applied as a strategy in targeted cancer treatment, where pharmaceutical inhibition of one gene combined with a mutation in its synthetic lethal partner gene in the tumor induces cancer cell death, while sparing healthy cells. An example of this is the FDA approved application of PARP inhibitors to treat breast cancer patients with a BRCA1 or BRCA2 mutation ${ }^{8}$. (Note, this gene pair would likely not have been detected with our method since it is based on induced essentiality: PARP mutations alone are not beneficial for the tumor and only become essential after mutation of BRCA1/2). Progress in other areas is however limited, often due to a poor understanding of the underlying mechanism, warranting further efforts to invest more in not only finding genetic interactions, but also understanding the biology behind them ${ }^{74}$.

Taken together, we show in our first map of genetic interactions in childhood cancer that cancer subtype, pathway epistasis and cooperation are the main underlying biological mechanisms that can explain our candidates, contributing each around 26 to $29 \%$ of all gene pairs. Only in $10 \%$ of the candidate gene pairs, synthetic lethality is the most likely explanation, while another $10 \%$ were most likely false positives due to technical artifacts. These percentages differ from expectations in most genetic interaction tests. Most tests aim to detect patterns of pathway epistasis or synthetic lethality in cancer but overlook the fact that other explanations play at least an equally important role. Studies into genetic interactions in cancer should therefore not stop at producing lists of candidate gene pairs but need to investigate what the most likely explanations are before drawing conclusions. The map also makes clear that these findings are the first steps towards exploring the full spectrum of genetic interactions in pediatric cancer and much more data is needed to understand the complete picture. 
1

2

3

4

5

6

7

8

9

\section{Methods}

\section{Data collection and processing}

To systematically detect genetic interactions in pediatric cancers, a collection of tumor samples from two recently published pediatric cancer studies was used in our pipeline. One study ${ }^{6}$ was carried out by the German Cancer Research Center (Deutsche Krebsforschungszentrum, DKFZ) while the second study is part of the Therapeutically Applicable Research to Generate Effective Treatments (TARGET) initiative ${ }^{7}$.

\section{DKFZ data set}

The DKFZ cohort covers 24 major childhood cancer types with an emphasis on central nervous system tumors (including atypical teratoid/rhabdoid tumors (ATRT); embryonal tumors with multilayered rosettes (ETMR); the four medulloblastoma groups WNT (MB-WNT), SSH (MB-SSH), Group3 (MB-GR3) and Group4 (MB-GR4), pilocytic astrocytoma (PA); high-grade glioma with and without histone 3 K27M mutations (HGG-K27M, HGG-other); infratentorial and supratentorial ependymoma (EPD-IT, EPD-ST)), hematological tumors (acute myeloid leukemia (AML); B-cell acute lymphoblastic leukemia with or without hypodiploidy (B-ALL-HYPO, B-ALL-other); T-cell acute lymphoblastic leukemia (T-ALL); Burkitt's lymphoma (BL)) as well as solid tumors (neuroblastoma (NB); Wilm's tumor (WT), osteosarcoma (OS); Ewing's sarcoma (EWS); hepatoblastoma (HB); adrenocortical carcinoma (ACT); rhabdomyosarcoma (RMS) and retinoblastoma (RB)). Note that to be able to compare results from both DKFZ and TARGET data sets, we merged B-ALL-HYPO and BALL-other samples into one B-ALL group.

The data set consists of 961 tumors (from 914 patients) sequenced with both paired and single end Illumina-based technology, including whole-genome sequences (WGS) and whole-exome sequences (WES). To avoid false positives, 82 relapse samples were excluded, as well as seven hypermutator samples (>ten coding mutations per Mb, see paragraph Hypermutator filtering) from the HGG-other cancer type (ICGC_GBM15, ICGC_GBM56, ICGC_GBM6, ICGC_GBM67, SJHGG030, SJHGG034, 
SJHGG111), and ten single-end sequenced samples (MB_Exm250, MB_Exm528, MB_Exm10,

Mutation data are available and can be downloaded from publicly available data portals such as http://pedpancan.com, but are limited to frequently mutated genes. In this study we aim to include all mutated genes and therefore use the high confident, but unfiltered results of the variant calling procedure described in Gröbner et al. ${ }^{6}$. In summary, raw FASTQ files were processed by the standardized alignment and variant calling pipeline developed by and applied in the ICGC Pan-Cancer project (https://github.com/ICGC-TCGA-PanCancer). The human genome assembly hs37d5 (ncbi.nlm.nih.gov/assembly/2758) was used as a reference genome and GENCODE19 (gencodegenes.org/releases/19.html) for gene annotation. Germline variants were determined based on their presence in the matched control tissue.

In cases where mutations were annotated to multiple genes, one of these genes was selected using a voting system based on annotation fields derived from Gencode version 19 (v19, used by DKFZ in their variant calling pipeline) and Gencode version 27 ( $v 27$, the most recent version at the time of doing the analyses). In this voting system, the first ranking gene was chosen after sorting the genes on the following properties: Gencode v19 status (KNOWN, NOVEL, PUTATIVE), Gencode v19 type (protein-coding, other), Gencode v27 type (protein-coding, other), total number of exonic alterations for this gene (higher numbers ranking higher), total number of exonic alterations in single genes (so alterations not overlapping other genes ranking higher) and gene name not containing '-' (which usually indicates a read-through gene).

Only somatic single nucleotide variants (SNVs) and small insertions and deletions (indels) were selected for this study and only those located in exonic regions of protein-coding genes that might have a functional consequence (frameshift, non-frameshift, non-synonymous, stopgain, stoploss) according to ANNOVAR annotation were included. In total, 9,922 SNVs and 1,236 indels remained for downstream analysis. Genes with at least one of the abovementioned mutations were considered as 
1

2

3

4

'mutated genes' and were kept for each sample. The final DKFZ data set that was used to identify genetic interactions includes 829 tumors, comprising 523 WGS and 306 WES samples. See Supplemental Table S1 for an overview of the consequences of each filtering step on tumor numbers in both data sets.

\section{TARGET data set}

The TARGET data set comprises of 1,699 primary tumors, of which 1,648 tumors with WGS or WES data (from 655 and 1,115 samples respectively) were available for download. Three tumors (10-PARTJ, 10-PANWIM, 30-PARJXH) were excluded as they could not be linked with corresponding meta data files. The remaining data set consists of six cancer types with most samples coming from hematological tumors (acute myeloid leukemia (AML, 208); B-cell acute lymphoblastic leukemia (B-ALL, 681); T-cell acute lymphoblastic leukemia (T-ALL, 267)) as well as solid tumors (neuroblastoma (NB, 286); osteosarcoma (OS, 88); Wilm's tumor (WT, 115)). MAF files with annotated somatic SNVs and indels for these cancer types were downloaded on March 21, 2018 (https://ocg.cancer.gov/programs/target/data-matrix). The variants listed in these files are the result of an initial variant calling pipeline using whole exome sequencing and Complete Genomics Inc. whole genome sequencing technology followed by thorough filtering as described in Ma et al. ${ }^{7}$.

Applying the same criteria as with the DKFZ set for hypermutators, one B-ALL tumor (10-PARBPX) and one NB tumor (30-PAPPKJ) were excluded from further analyses. From the remaining 1,643 tumors a total of 19,865 SNVs and 2,272 indels located in exons and likely having a functional effect (annotated as Missense_Mutation, Nonsense_Mutation, missense, nonsense, Frame_Shift_Del, Frame_Shift_Ins, In_Frame_Del, In_Frame_Ins, frameshift, proteinDel, proteinIns) were used to detect genetic interactions. Eleven tumors did not contain such functional alterations, resulting in a total of 1,632 tumors (649 WGS samples and 1,105 WES samples) that were included in downstream analyses. 
1

2

3

4

5

\section{Hypermutator filtering}

Hypermutators are usually defined as tumors with more than ten coding mutations per Mb, but there is currently no consensus about what type of mutations and which genomic region should be included to define a hypermutator ${ }^{75}$. In this study, where we test two data sets from different sources, we decided to apply the threshold of ten mutations per $\mathrm{Mb}$ on a strictly defined coding region of known length and counting the number of mutations in this region including all SNVs (missense, nonsense and silent) and all indels. We used the GeneBase tool ${ }^{76}$ to extract the nonredundant length of the coding part of exons in a set of protein-coding genes from the NCBI database where the gene and its transcripts have status REVIEWED/VALIDATED. The exact non-redundant length of the coding part of these 18,255 genes is $23,698,355 \mathrm{bp}(23.7 \mathrm{Mb})$. Assuming that the sequencing reads in our data sets have at least $95 \%$ sufficient coverage in this region, so having 22.5 $\mathrm{Mb}$ or more covered, each sample with more than 225 coding mutations in this set of genes will be considered a hypermutator.

\section{Genetic interaction analyses}

\section{Permutation test}

To detect significant cases of co-occurrence and mutual exclusivity, we followed a permutation approach similar as described $\mathrm{in}^{23}$. First, for each cancer type, we constructed binary mutation matrices, recording per sample if it has one or more mutations (SNVs or indels) in a certain gene (Supplemental Figure S5). Genes with only one mutation in a mutation matrix were excluded to reduce computational time. For each gene pair we counted the number of co-occurrences, that is the number of samples that have both genes mutated. Secondly, we used the Permatswap function in the vegan $\mathrm{R}$ library (version $2.5-4$, using default parameters, except for $m$ type $=$ "prab") to generate a series of permuted matrices $\left(N=1 \times 10^{6}\right)$, while keeping their margins fixed. In other words, with each permutation the total number of mutated genes per gene and per sample still match with the 
original mutation matrix. Next, an empirical $P$-value for co-occurrence $\left(P_{\mathrm{co}}\right)$ was calculated for each pair of mutated genes by taking the proportion of permutations in which the co-occurrence count was equal to or higher than the observed count. To be more specific, the $P_{\text {co }}$ value for a gene pair $\left(g_{1}, g_{2}\right)$ was calculated as follows:

$$
P_{c o}\left(g_{1}, g_{2}\right)=\frac{1+\sum_{i=1}^{N}\left[\mathrm{co}_{i}\left(g_{1}, g_{2}\right) \geq \mathrm{co}_{o b s}\left(g_{1}, g_{2}\right)\right]}{1+N}
$$

where $N$ is the number of permutations and $\operatorname{co}_{o b s}\left(g_{1}, g_{2}\right)$ and $\mathrm{co}_{i}\left(g_{1}, g_{2}\right)$ represent the cooccurrence count for genes $g_{1}$ and $g_{2}$ in the observed and $i^{\text {th }}$ permuted matrix respectively. Note that we add a one in both the numerator and denominator to have a good estimator of the $P$-value and to avoid $P$-values of zero ${ }^{77}$. In a similar fashion, a $P$-value for mutual exclusivity $\left(P_{\text {me }}\right)$ was calculated as the proportion of permutations in which the co-occurrence count was equal to or lower than the observed data.

As the empirical $P$-values were not uniformly distributed and showed a bias towards one, we could not use standard false discovery rate (FDR) calculations to correct for multiple hypothesis testing. Instead, we estimated the FDR empirically by creating a $P$-value null distribution. We randomly selected 100 matrices from the permuted matrices and performed the genetic interaction permutation test on each matrix to generate a random set of $P$-values, $\mathrm{S}_{\text {null }}$ (Fig detailed workflow test). For each observed $P$-value $P^{*}$ (computed in the previous step), the FDR was estimated as follows (if all hypotheses with a $P$-value $\leq P^{*}$ would be rejected):

$$
F D R\left(P^{*}\right)=\frac{V\left(P^{*}\right)}{R\left(P^{*}\right)}
$$

Where $V\left(P^{*}\right)$ is the estimated number of false positives and $R\left(P^{*}\right)$ is the total number of rejected null hypotheses. $V\left(P^{*}\right)$ was estimated from the proportion of $P$-values $\leq P^{*}$ in $\mathrm{S}_{\text {null }}$ multiplied by the total number of observed $P$-values. Finally, a Q-value was determined by taking the lowest estimated FDR among all observed $P$-values $\geq P^{*}$. Gene pairs were considered as significant if they scored a Q- 

occurring gene-pairs.

3

\section{WeSME test}

In the pan-cancer analysis, we performed the same test on a mutation matrix constructed with all cancer types combined, but permutations were carried out for each cancer type separately to control for any biases in mutation frequencies.

As the matrix permutation approach is rather time consuming, we also applied a faster genetic interaction test and compared the results of both tests to infer their overlap. This test, called Weighted Sampling based Mutual Exclusivity (WeSME), starts similar to the permutation approach with a gene-sample mutation matrix from which for each gene pair the number of mutual exclusive samples is counted ${ }^{28}$. Instead of permuting this matrix many times to compute a $P$-value, WeSME uses a weighted sampling approach based on the mutation rate of the samples. The method also reduces computation time by restricting the number of resamplings as it starts with a small null distribution and only increases the number of resamplings (to a maximum of $N=10,000$ ) for candidate gene pairs, namely those that have a low $P$-value estimated from the initial null distribution.

To infer the false discovery rate, a similar empirical FDR approach is used as with the matrix permutation method, by permuting the mutation matrix 300 times and applying the WeSME test on the permuted matrices to create a $P$-value null distribution. WeSME places genes in either of two mutation rate bins 'high' and 'low' (with 2\% of the samples being mutated as threshold) and compares the observed $P$-values with a $P$-value distribution of gene pairs from similar bins. The null $P$-values are thus split into three distributions representing the combinations of mutation rate bins ([low,low], [low, high] and [high,high]). Since null P-values are assigned to three different bins, 300 permutations were performed instead of 100 as was done in the permutation test. The WeSME Python scripts were downloaded from 
suitable for the current study. In particular, adaptions to the original scripts were made to run a pancancer analysis by pooling data from multiple cancer types, counting mutual exclusivity over the pooled set, but performing resampling within each cancer type. To reduce computational time in the PAN cancer test, gene-pairs were only tested for mutual exclusivity if the total number of mutually exclusive samples was at least three. With the co-occurrence test, at least one sample needed to have a co-occurring mutated gene pair. In contrast to the original WeSME code, the $P$-values of all gene-pairs were used for FDR calculations instead of only keeping gene-pairs with $P$-values below 0.1. As in the Permutation test, only gene pairs with an empirical FDR $<0.2$, a $P$-value $<0.1$ and a minimum co-occurrence count of three (only when testing for co-occurring gene-pairs) were considered as significant candidates for further analysis.

To avoid potential biases due to this sampling-based method, we repeated the analyses ten times, each time using another randomization seed and considered only as high confidence candidates those gene pairs that scored significant in at least nine out of ten tests. Note that the Permutation Test is also based on resampling but was only run once because of its higher computation time.

\section{Mutation load analyses}

For each gene that was part of a candidate gene pair, the mutation load association (MLA) were calculated following ${ }^{27}$. In short, we used the R function glm from the stats package to run a logistic regression of mutation frequency on mutation load and calculated the MLA score by dividing the regression coefficient by the standard error. Both the mutation frequency (of a gene) and mutation load (of a sample) were calculated from the column and row sums respectively of the sample-gene mutation matrix used in our genetic interaction test. All candidate gene pairs with an MLA difference larger than three and at least one gene with an MLA larger than three were marked as 'suspect'. 
2 T-ALL subtype annotations were downloaded from the St. Jude's PeCan Portal

3 (https://pecan.stjude.cloud/proteinpaint/study/target-tall) which displays mutation data of T-ALL

4 tumors in a large genomics study ${ }^{31}$. Of all 266 T-ALL samples used in our data set, we could assign

5263 tumors a subtype category (Ordered by maturation stage: LMO2/LYL1: 18, HOXA: 33, TLX3: 46,

6 TLX1: 26, NKX2-1: 14, LMO1/2: 10, TAL1: 86, TAL2: 8, Unknown: 22). We removed the three

7 unassigned tumors, and those from the 'unknown' category before generating Figure 5.

\section{Candidate Reporting web application}

10 We developed an R (v3.5.2) web application with Shiny (v1.5.0) to provide visualizations and additional up-to-date variant annotation of genetic interaction candidates. We first ran the Ensembl Variant Effect Predictor (VEP) ${ }^{78}$ GRCh37 (V101) on the original set of mutations to predict the genes, transcripts, protein sequences and their consequences. We restricted results by selecting VEP fields indicating the impact of the variant (e.g. Consequence, Condel) and by choosing one consequence per variant, which translates to the top-ranking transcript. In addition, fields indicating the cancer type and the source data set were added to the VEP output and the final table was translated to a Mutation Annotation Format (MAF) object. Maftools ${ }^{79}$ (v1.8.10) was used to create lollipop plots, oncoplots and data summary plots from subsets of the MAF object by filtering on the cancer type and data set. One-letter code amino acid changes for the lollipop plots were derived from the protein sequence in HGVS recommended format (HGVSp field of VEP). The Candidate Reporting web application can be found on http://gi-analysis.bioinf.prinsesmaximacentrum.nl/ (available as of December 11, 2020).

After running VEP annotation for the reporting tool, we noticed minor changes in gene annotation in candidate gene pairs compared to the original mutation files. Six patients (three in T-ALL, one in AML and one in B-ALL) had mutations previously annotated in CNKSR3, while these were actually 
mutations in the gene MAGI1. This affected all (three) T-ALL patients that were involved in the mutually exclusive candidate CNKSR3-NOTCH1, and we therefore changed this candidate in MAGI1NOTCH1. Furthermore, we found three patients (one in T-ALL and two in NBL) with mutations previously annotated in PTEN where these should be mutations in TEP1. PTEN is part of several T-ALL candidate genetic interactions. We therefore repeated the WeSME test in T-ALL ( $N=20$ runs), changing this one patient mutation from PTEN to TEP1. Some interactions (NOTCH1-USP7, PHGSPIK3R1, PHF6-PTEN and USP7-WT1) scored lower with significance in 17 out of 20 runs (compared to significance in 9 or 10 out of 10 runs previously). As these are still high confidence candidates, we kept them in our final result set.

\section{Data availability}

The somatic mutation data that support our findings are available via the National Cancer Institute TARGET Data Matrix (https://ocg.cancer.gov/programs/target/data-matrix) and the R2 DKFZ Pediatric Cancer Data Portal (https://hgserver1.amc.nl/cgi-bin/r2/main.cgi?option=about dscope). We further refer to the original studies ${ }^{6,7}$ for more information on data availability of the raw data files. Processed data files that served as input for our genetic interaction pipeline (gene-sample mutation matrices) are available upon request.

\section{Used software and Code availability}

Figure 3 was created with the network visualization software Cytoscape (version 3.7.1).

$\mathrm{R}$ code to run the Permutation test and the modified WeSME Python scripts are available on the Github repository https://github.com/princessmaximacenter/GI InteractionTests (available as of December 11, 2020). The code for the R Shiny Candidate Reporting tool is available on https://github.com/princessmaximacenter/Gl ReportingTool (available as of December 1, 2020). 
1. Siegel, R. L., Miller, K. D. \& Jemal, A. Cancer statistics, 2019. CA. Cancer J. Clin. 69, 7-34 (2019).

2. Oeffinger, K. C. et al. Chronic health conditions in adult survivors of childhood cancer. N. Engl. J. Med. 355, 1572-1582 (2006).

3. Bhatia, S. et al. Collaborative Research in Childhood Cancer Survivorship: The Current Landscape.

4. Vogelstein, B. et al. Cancer Genome Landscapes. Science 339, 1546-1558 (2013).

5. Downing, J. R. et al. The Pediatric Cancer Genome Project. Nat. Genet. 44, 619-622 (2012).

6. Gröbner, S. N. et al. The landscape of genomic alterations across childhood cancers. Nature (2018) doi:10.1038/nature25480.

7. Ma, X. et al. Pan-cancer genome and transcriptome analyses of 1,699 paediatric leukaemias and solid tumours. Nature (2018) doi:10.1038/nature25795.

8. Ashworth, A., Lord, C. J. \& Reis-Filho, J. S. Genetic Interactions in Cancer Progression and Treatment. Cell 145, 30-38 (2011).

9. Lehner, B., Crombie, C., Tischler, J., Fortunato, A. \& Fraser, A. G. Systematic mapping of genetic interactions in Caenorhabditis elegans identifies common modifiers of diverse signaling pathways. Nat. Genet. 38, 896-903 (2006).

10. Huang, W. et al. Epistasis dominates the genetic architecture of Drosophila quantitative traits. Proc. Natl. Acad. Sci. 109, 15553-15559 (2012).

11. Costanzo, M. et al. A global genetic interaction network maps a wiring diagram of cellular function. Science 353, aaf1420 (2016).

12. Srivas, R. et al. A Network of Conserved Synthetic Lethal Interactions for Exploration of Precision Cancer Therapy. Mol. Cell 63, 514-525 (2016).

13. Laufer, C., Fischer, B., Billmann, M., Huber, W. \& Boutros, M. Mapping genetic interactions in human cancer cells with RNAi and multiparametric phenotyping. Nat. Methods 10, 427-431 (2013). 
14. Najm, F. J. et al. Orthologous CRISPR-Cas9 enzymes for combinatorial genetic screens. Nat. Biotechnol. 36, 179 (2017).

15. Boettcher, M. et al. Dual gene activation and knockout screen reveals directional dependencies in genetic networks. Nat. Biotechnol. 36, 170 (2018).

16. Dobzhansky, Th. Genetics of Natural Populations. Xiii. Recombination and Variability in Populations of Drosophila Pseudoobscura. Genetics 31, 269-290 (1946).

17. Fong, P. C. et al. Inhibition of Poly(ADP-Ribose) Polymerase in Tumors from BRCA Mutation Carriers. N. Engl. J. Med. 361, 123-134 (2009).

18. Hartwell, L. H., Szankasi, P., Roberts, C. J., Murray, A. W. \& Friend, S. H. Integrating Genetic Approaches into the Discovery of Anticancer Drugs. Science 278, 1064-1068 (1997).

19. Shen, J. P. \& Ideker, T. Synthetic Lethal Networks for Precision Oncology: Promises and Pitfalls. J. Mol. Biol. 430, 2900-2912 (2018).

20. O’Neil, N. J., Bailey, M. L. \& Hieter, P. Synthetic lethality and cancer. Nat. Rev. Genet. 18, 613623 (2017).

21. Ciriello, G., Cerami, E., Sander, C. \& Schultz, N. Mutual exclusivity analysis identifies oncogenic network modules. Genome Res. 22, 398-406 (2012).

22. Leiserson, M. D. M., Blokh, D., Sharan, R. \& Raphael, B. J. Simultaneous Identification of Multiple Driver Pathways in Cancer. PLOS Comput. Biol. 9, e1003054 (2013).

23. Park, S. \& Lehner, B. Cancer type-dependent genetic interactions between cancer driver alterations indicate plasticity of epistasis across cell types. Mol. Syst. Biol. 11, (2015).

24. Mina, M. et al. Conditional Selection of Genomic Alterations Dictates Cancer Evolution and Oncogenic Dependencies. Cancer Cell (2017) doi:10.1016/j.ccell.2017.06.010.

25. Deng, Y. et al. Identifying mutual exclusivity across cancer genomes: computational approaches to discover genetic interaction and reveal tumor vulnerability. Brief. Bioinform. 20, 254-266 (2019). 
26. Canisius, S., Martens, J. W. M. \& Wessels, L. F. A. A novel independence test for somatic alterations in cancer shows that biology drives mutual exclusivity but chance explains most cooccurrence. Genome Biol. 17, 261 (2016).

27. van de Haar, J. et al. Identifying Epistasis in Cancer Genomes: A Delicate Affair. Cell 177, 13751383 (2019).

28. Kim, Y.-A., Madan, S. \& Przytycka, T. M. WeSME: uncovering mutual exclusivity of cancer drivers and beyond. Bioinformatics 33, 814-821 (2017).

29. Meijerink, J. P. P. Genetic rearrangements in relation to immunophenotype and outcome in Tcell acute lymphoblastic leukaemia. Best Pract. Res. Clin. Haematol. 23, 307-318 (2010).

30. Belver, L. \& Ferrando, A. The genetics and mechanisms of T cell acute lymphoblastic leukaemia. Nat. Rev. Cancer 16, 494-507 (2016).

31. Liu, Y. et al. THE GENOMIC LANDSCAPE OF PEDIATRIC AND YOUNG ADULT T-LINEAGE ACUTE LYMPHOBLASTIC LEUKEMIA. Nat. Genet. 49, 1211-1218 (2017).

32. Wu, G. et al. The genomic landscape of diffuse intrinsic pontine glioma and pediatric nonbrainstem high-grade glioma. Nat. Genet. 46, 444-450 (2014).

33. Castel, D. et al. Histone H3F3A and HIST1H3B K27M mutations define two subgroups of diffuse intrinsic pontine gliomas with different prognosis and phenotypes. Acta Neuropathol. (Berl.) 130, 815-827 (2015).

34. Castel, D. et al. Transcriptomic and epigenetic profiling of 'diffuse midline gliomas, H3 K27Mmutant' discriminate two subgroups based on the type of histone $\mathrm{H} 3$ mutated and not supratentorial or infratentorial location. Acta Neuropathol. Commun. 6, 117 (2018).

35. Northcott, P. A. et al. The whole-genome landscape of medulloblastoma subtypes. Nature 547, 311 (2017).

36. Kool, M. et al. Genome Sequencing of SHH Medulloblastoma Predicts Genotype-Related Response to Smoothened Inhibition. Cancer Cell 25, 393-405 (2014). 
37. Scholz, H. \& Kirschner, K. M. Oxygen-Dependent Gene Expression in Development and Cancer: Lessons Learned from the Wilms' Tumor Gene, WT1. Front. Mol. Neurosci. 4, (2011).

38. Yang, L., Han, Y., Saurez Saiz, F. \& Minden, M. D. A tumor suppressor and oncogene: the WT1 story. Leukemia $\mathbf{2 1}, 868-876$ (2007).

39. Schwartzentruber, J. et al. Driver mutations in histone H3.3 and chromatin remodelling genes in paediatric glioblastoma. Nature 482, 226-231 (2012).

40. Fortin, J. et al. Mutant ACVR1 Arrests Glial Cell Differentiation to Drive Tumorigenesis in Pediatric Gliomas. Cancer Cell 37, 308-323.e12 (2020).

41. Pathak, P. et al. Altered global histone-trimethylation code and H3F3A-ATRX mutation in pediatric GBM. J. Neurooncol. 121, 489-497 (2015).

42. Nandakumar, P., Mansouri, A. \& Das, S. The Role of ATRX in Glioma Biology. Front. Oncol. 7, (2017).

43. Froimchuk, E., Jang, Y. \& Ge, K. Histone H3 lysine 4 methyltransferase KMT2D. Gene 627, 337342 (2017).

44. Shi, X. et al. An epigenetic switch induced by Shh signalling regulates gene activation during development and medulloblastoma growth. Nat. Commun. 5, 5425 (2014).

45. Roussel, M. F. \& Stripay, J. L. Epigenetic Drivers in Pediatric Medulloblastoma. The Cerebellum 17, 28-36 (2018).

46. Larson, J. D. et al. Histone H3.3 K27M Accelerates Spontaneous Brainstem Glioma and Drives Restricted Changes in Bivalent Gene Expression. Cancer Cell 35, 140-155.e7 (2019).

47. Singh, B. et al. p53 regulates cell survival by inhibiting PIK3CA in squamous cell carcinomas. Genes Dev. 16, 984-993 (2002).

48. Maxson, J. E. et al. CSF3R mutations have a high degree of overlap with CEBPA mutations in pediatric AML. Blood 127, 3094-3098 (2016).

49. Braun, T. P. et al. Myeloid lineage enhancers drive oncogene synergy in CEBPA/CSF3R mutant acute myeloid leukemia. Nat. Commun. 10, 5455 (2019). 
50. Tien, F.-M. et al. Concomitant WT1 mutations predict poor prognosis in acute myeloid leukemia patients with double mutant CEBPA. Haematologica 103, e510-e513 (2018).

51. Wilhelmson, A. S. \& Porse, B. T. CCAAT enhancer binding protein alpha (CEBPA) biallelic acute myeloid leukaemia: cooperating lesions, molecular mechanisms and clinical relevance. Br. J. Haematol. 190, 495-507 (2020).

52. Kats, L. M. et al. Proto-Oncogenic Role of Mutant IDH2 in Leukemia Initiation and Maintenance. Cell Stem Cell 14, 329-341 (2014).

53. Ogawara, Y. et al. IDH2 and NPM1 Mutations Cooperate to Activate Hoxa9/Meis1 and Hypoxia Pathways in Acute Myeloid Leukemia. Cancer Res. 75, 2005-2016 (2015).

54. Grafone, T., Palmisano, M., Nicci, C. \& Storti, S. An overview on the role of FLT3-tyrosine kinase receptor in acute myeloid leukemia: biology and treatment. Oncol. Rev. 6, (2012).

55. Leiserson, M. D. M., Wu, H.-T., Vandin, F. \& Raphael, B. J. CoMEt: a statistical approach to identify combinations of mutually exclusive alterations in cancer. Genome Biol. 16, 160 (2015).

56. Vicent, S. et al. Wilms tumor 1 (WT1) regulates KRAS-driven oncogenesis and senescence in mouse and human models. J. Clin. Invest. 120, 3940-3952 (2010).

57. Woo, J. S., Alberti, M. O. \& Tirado, C. A. Childhood B-acute lymphoblastic leukemia: a genetic update. Exp. Hematol. Oncol. 3, 16 (2014).

58. Paulsson, K. et al. Mutations of FLT3, NRAS, KRAS, and PTPN11 are frequent and possibly mutually exclusive in high hyperdiploid childhood acute lymphoblastic leukemia. Genes. Chromosomes Cancer 47, 26-33 (2008).

59. Aguirre, A. J. \& Hahn, W. C. Synthetic Lethal Vulnerabilities in KRAS-mutant Cancers. Cold Spring Harb. Perspect. Med. 8, (2018).

60. Springuel, L. et al. Cooperating JAK1 and JAK3 mutants increase resistance to JAK inhibitors. Blood 124, 3924-3931 (2014).

61. Tavakoli Shirazi, P. et al. The effect of co-occurring lesions on leukaemogenesis and drug response in T-ALL and ETP-ALL. Br. J. Cancer 122, 455-464 (2020). 
62. Zuurbier, L. et al. NOTCH1 and/or FBXW7 mutations predict for initial good prednisone response but not for improved outcome in pediatric T-cell acute lymphoblastic leukemia patients treated

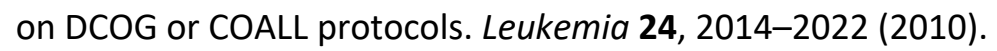

63. Zuurbier, L. et al. The significance of PTEN and AKT aberrations in pediatric T-cell acute lymphoblastic leukemia. Haematologica 97, 1405-1413 (2012).

64. Canté-Barrett, K. et al. MEK and PI3K-AKT inhibitors synergistically block activated IL7 receptor signaling in T-cell acute lymphoblastic leukemia. Leukemia 30, 1832-1843 (2016).

65. Morrison, D. J., Kim, M. K., Berkofsky-Fessler, W. \& Licht, J. D. WT1 Induction of MAP Kinase Phosphatase 3 Represents a Novel Mechanism of Growth Suppression. Mol. Cancer Res. MCR 6, $1225-1231$ (2008).

66. Kozakai, T. et al. MAGI-1 expression is decreased in several types of human T-cell leukemia cell lines, including adult T-cell leukemia. Int. J. Hematol. 107, 337-344 (2018).

67. Jin, Q. et al. USP7 cooperates with NOTCH1 to drive the oncogenic transcriptional program in T cell leukemia. Clin. Cancer Res. clincanres.1740.2018 (2018) doi:10.1158/1078-0432.CCR-181740.

68. Prat, J., Gray, G. F., Stolley, P. D. \& Coleman, J. W. Wilms Tumor in an Adult Associated With Androgen Abuse. JAMA 237, 2322-2323 (1977).

69. Gadd, S. et al. A Children's Oncology Group and TARGET initiative exploring the genetic landscape of Wilms tumor. Nat. Genet. 49, 1487-1494 (2017).

70. Nikolaev, S. I. et al. Frequent cases of RAS-mutated Down syndrome acute lymphoblastic leukaemia lack JAK2 mutations. Nat. Commun. 5, 4654 (2014).

71. The AACR Project GENIE Consortium. AACR Project GENIE: Powering Precision Medicine through an International Consortium. Cancer Discov. 7, 818-831 (2017).

72. Ijaz, H. et al. Pediatric high-grade glioma resources from the Children's Brain Tumor Tissue Consortium. Neuro-Oncol. 22, 163-165 (2020). 
73. McLeod, C. et al. St. Jude Cloud-a Pediatric Cancer Genomic Data Sharing Ecosystem. bioRxiv 2020.08.24.264614 (2020) doi:10.1101/2020.08.24.264614.

74. Tutuncuoglu, B. \& Krogan, N. J. Mapping genetic interactions in cancer: a road to rational combination therapies. Genome Med. 11, 62 (2019).

75. Campbell, B. B. et al. Comprehensive Analysis of Hypermutation in Human Cancer. Cell 171, 1042-1056.e10 (2017).

76. Piovesan, A., Caracausi, M., Antonaros, F., Pelleri, M. C. \& Vitale, L. GeneBase 1.1: a tool to summarize data from NCBI gene datasets and its application to an update of human gene statistics. Database J. Biol. Databases Curation 2016, (2016).

77. Phipson, B. \& Smyth, G. K. Permutation P-values Should Never Be Zero: Calculating Exact Pvalues When Permutations Are Randomly Drawn. Stat. Appl. Genet. Mol. Biol. 9, (2010).

78. McLaren, W. et al. The Ensembl Variant Effect Predictor. Genome Biol. 17, 122 (2016). comprehensive analysis of somatic variants in cancer. Genome Res. 28, 1747-1756 (2018). 
1

\section{Acknowledgements}

2 We would like to thank Jarno Drost, Kim Verhagen, Jules Meijerink, Esther Hulleman, Judith

3 Boer, Frank van Leeuwen and members of the lab for fruitful discussions and feedback.

4 Financial support has been provided by the Dutch Cancer Society (grant number 10354), as

5 well as by the Dutch Organisation for Scientific Research (NWO; grant number 864.11.010)

6 and KiKa.

7

\section{Author Contributions}

$9 \quad \mathrm{PK}, \mathrm{FH}$ and JD designed the study. JD and SA designed and implemented the statistical pipeline and analyzed and interpreted test results. DK designed and implemented the candidate reporting tool. SM, NJ, JZ, SP provided the data set and assisted in interpretation of the data.

JD, SA, PK and DK prepared the manuscript. JZ, SP and FH edited the manuscript.

\section{Competing interests}

15 The authors declare no competing interests. 